\title{
Fish Protein Promotes Skeletal Muscle Hypertrophy via the Akt/mTOR Signaling Pathways
}

\author{
Mizuki MorIsASA ${ }^{1}$, Eriko YoshidA ${ }^{2}$, Mina FujITANI ${ }^{3, *}$, Keisuke KImURA ${ }^{1}$, Kenji UCHIDA ${ }^{2}$, \\ Taro KISHIDA ${ }^{3}$, Tsukasa MORI ${ }^{1}$ and Naoko GoTO-INOuE ${ }^{1, * *}$ \\ ${ }^{1}$ Department of Marine Science and Resources, College of Bioresource Sciences, Nihon University, \\ 1866 Kameino, Fujisawa, Kanagawa 252-0880, Japan \\ ${ }^{2}$ Functional Ingredient Research Section, Food Function RED Center, Nippon Suisan Kaisha, Ltd., \\ Tokyo 105-8676, Japan \\ ${ }^{3}$ Laboratory of Nutrition Science, Division of Applied Bioscience, Graduate School of Agriculture, \\ Ehime University, Matsuyama, Ehime 790-8566, Japan
}

(Received July 5, 2021)

\begin{abstract}
Summary Skeletal muscle is the largest organ in the body and has a broad range of plasticity, undergoing atrophy in response to aging or disease and hypertrophy in response to nutritional supplements or exercise. Loss of skeletal muscle mass and force increases the risk of falls, impairs mobility, and leads to reduced quality of life. In a previous study, we demonstrated that taking in Alaska pollock protein (APP) for only $7 \mathrm{~d}$ increased the gastrocnemius muscle mass in rats. This study was conducted to identify hypertrophic myofibers and analyze how hypertrophy occurs within them. Twenty male rats were randomly divided into two groups and administered a diet of casein or APP for $7 \mathrm{~d}$. The expression of each myosin heavy chain (MyHC) isoform in a cross-sectional area was then measured. $\mathrm{MyHC} \mathrm{IIb} \mathrm{and} \mathrm{IIx} \mathrm{isoforms} \mathrm{exhibited} \mathrm{hypertrophic} \mathrm{features} \mathrm{in} \mathrm{the} \mathrm{gastrocnemius} \mathrm{muscles} \mathrm{of}$ the APP-fed rats. Furthermore, comprehensive proteomic analyses were conducted to identify changes in protein expression due to muscle hypertrophy. Our results, evaluated by pathway analyses, indicated that the activity of the growth factor signaling pathway was significantly impacted by APP consumption. Moreover, APP could promote protein synthesis by activating the protein kinase B/mechanistic target of the rapamycin signaling pathway, which is also promoted by exercise.
\end{abstract}

Key Words Alaska pollock protein, skeletal muscle, hypertrophy, Akt/mTOR signaling, myosin fiber type

Skeletal muscle is the most extensive muscular system in the human body, accounting for approximately $40 \%$ of the total body weight. It is responsible for normal movement and imparts tensile strength, and a balance between protein degradation and synthesis is required to maintain skeletal muscle mass. Therefore, changes in protein degradation and/or synthesis can cause skeletal muscle atrophy and hypertrophy. Skeletal muscle consists of two types of muscle fibers: slowtwitch (type I) and fast-twitch (type II), which are heterogeneously distributed in the muscle. The rate of muscle loss with aging is approximately $1-2 \%$ per year past the age of $50(1,2)$; in particular, the amount of fasttwitch fiber tends to decrease (3). Skeletal muscle mass

\footnotetext{
*,**To whom correspondence should be addressed.

*E-mail: fujitani.mina.uu@ehime-u.ac.jp

**E-mail: inoue.naoko@nihon-u.ac.jp

Abbreviations: Akt, protein kinase B; APP, Alaska pollack protein; Atrogin1, F-box protein 32; CSA, cross-sectional area; FOXO, Forkhead box O; IGF, insulin-like growth factor 1; IPA, ingenuity pathway analysis; mTOR, mechanistic target of the rapamycin; MuRF1, muscle-specific RING finger protein 1 ; MyHC, myosin heavy chain; Myostatin, growth differentiation factor 8 .
}

and strength should be maintained to prevent age-related muscle disorders, which can be achieved by the activation of protein synthesis pathways in muscle cells. Several studies have proposed that resistance training, dietary remedies, and nutritional supplements may serve as effective treatment strategies for age-related muscle disorders $(4,5)$. Various reports have explored muscle hypertrophy via nutritional supplements. A previous study reported that oral lactate administration increased the tibialis anterior muscle weight and fiber cross-sectional area (CSA) in mice (6), and another indicated that taking in leucine along with exercise can activate protein synthesis signaling in muscle cells (7). Multiple studies have reported the importance of boosting muscle mass and maintaining muscle strength by administering a protein-rich $\operatorname{diet}(8)$.

Herein, we studied the ability of fish proteins to promote muscle hypertrophy. Fish consumption has risen worldwide in recent years, and several studies have highlighted the beneficial effects of fish proteins $(9,10)$. Moreover, the nutritional benefits of fish proteins should be determined because the edible portion of fish is rich in proteins and low in fat. Alaska pollock (Theragra chalcogramma) is an integral component of processed sea- 
food products, such as surimi, fish sausages, and kamaboko. We previously reported that Alaska pollock protein (APP) intake can increase the muscle mass and decrease the serum triglyceride levels in rats $(11,12)$. Importantly, these effects were not attributed to fish oil, because APP was eliminated from fish oil. Our previous study demonstrated a significant increase in the weight of hypertrophic gastrocnemius muscle in APP-fed rats after $7 \mathrm{~d}$ without exercise training (13). However, we did not mention "where" (which type of myofibers) hypertrophic features were observed and "how" hypertrophy occurred.

In this study, we investigated the mechanism of APP-induced muscle hypertrophy. Muscle hypertrophy can be induced by the promotion of the synthesis pathway and/or the suppression of the degradation pathway. Physical exercise and nutrient anabolism can upregulate insulin-like growth factor 1 (IGF-1) upstream of Akt/mTOR signaling and promote protein synthesis (14). In contrast, the Forkhead box O (FOXO)-dependent ubiquitin-proteasome system promotes protein degradation by augmenting the expression of ligase F-box protein 32 (Atrogin1) and the muscle-specific RING finger protein 1 (MuRF1) (15-17). In our previous work, we reported the effect of APP on canceling the atrophic features of the immobilization of the limbs, but we do not know the effect of APP in normal situation (18). We hypothesized that APP consumption may be linked to muscle hypertrophy under normal conditions in different pathway with immobilization. We used iTRAQ-based quantitative proteomic analysis to detect over 20,000 peptides in the gastrocnemius muscle in order to identify the pathway that may be affected and demonstrate how APP causes hypertrophy.

\section{MATERIALS AND METHODS}

Materials. As described in our previous study, male Sprague-Dawley rats aged $5 \mathrm{wk}$ were purchased from Japan SLC, Inc. (Shizuoka, Japan). All rats were housed in stainless-steel wire mesh cages in a temperature-controlled room $\left(23 \pm 1^{\circ} \mathrm{C}\right)$ under a 12-h light/dark cycle (dark phase: 15:00-3:00) (18). At 6 wk of age, the animals were assigned to one of two experimental groups: the casein diet group, which was fed an AIN-93 diet with casein as the protein source, or the APP group, which was fed a diet containing APP in place of casein (11). The rats were housed separately for $5 \mathrm{~d}$ to acclimate to the environment throughout the 7 -d feeding experiment with casein and APP. After $7 \mathrm{~d}$ of feeding, the rats were sacrificed and the gastrocnemius muscle was excised. This study was conducted in accordance with the ethical guidelines of the Ehime University Animal Experimentation Committee (08A92) and was in complete compliance with the National Institutes of Health: Guide for the Care and Use of Laboratory Animals. All efforts were made to minimize the number of animals used and limit experimentation to what was necessary in order to generate reliable scientific information. These experiments were conducted in September 2018.
Sample preparation. Following decapitation, the soleus, gastrocnemius, and extensor digitorum longus (EDL) muscles were collected on the final day of the experiment along with a non-fasting blood sample. The muscles were frozen in liquid nitrogen-cooled isopentane and stored at $-80^{\circ} \mathrm{C}$. Approximately $50 \mathrm{mg}$ of the gastrocnemius muscles were cut into pieces and homogenized after adding $500 \mu \mathrm{L}$ of a lysis buffer to extract total protein. After sonication on ice, the lysate was centrifuged at $10,000 \times g$ for $40 \mathrm{~min}$ at $4^{\circ} \mathrm{C}$ to form pellets. The total protein concentration of the supernatant was determined using a BCA Protein Assay kit (Pierce, Thermo Scientific, Waltham, MA, USA). The supernatant sample was stored at $-30^{\circ} \mathrm{C}$, and the blood samples were centrifuged at $1,500 \times g$ and $4^{\circ} \mathrm{C}$ for $10 \mathrm{~min}$ and stored at $-60^{\circ} \mathrm{C}$ until analysis.

Immunohistochemical staining to determine the myosin fiber type. Frozen gastrocnemius muscles were cut at the middle of the tissues, trimmed 10-20 times to $40-\mu \mathrm{m}$ thickness. Serial $10 \mu \mathrm{m}$-thick cross-sections of muscles were prepared using a cryostat (CM 1950; Leica Microsystems, Wetzlar, Germany) and mounted on Matsunami Adhesive-coated slides (Matsunami, Osaka, Japan). To differentiate each muscle fiber type, the muscle sections were stained to identify fiber typespecific antibodies (19). The acetone-fixed cryosections were washed thrice with phosphate-buffered saline containing Tween (PBST), blocked with Blocking One Histo (Nacalai Tesque, Inc., Tokyo, Japan) for $20 \mathrm{~min}$, and incubated overnight at $4^{\circ} \mathrm{C}$ with primary antibodies against MyHC I (\#BA-F8), MyHC IIa (\#SC-71), and MyHC IIb (\#BF-F3), which were all obtained from the Development Studies Hybridoma Bank, University of Iowa. Thereafter, the tissue sections were washed five times with PBST and incubated for $1 \mathrm{~h}$ at $23^{\circ} \mathrm{C}$ with secondary antibodies conjugated to Alexa Fluor 350 (\#A21040), Alexa Fluor 488 (\#A21042), and Alexa Fluor 555 (\#A21127), which were all purchased from Thermo Fisher Scientific KK, Tokyo, Japan. The fluorescence intensity was detected using a wide-field fluorescence microscope (BZ-9000; Keyence, Osaka, Japan). CSA of each myofiber was measured in three independent animals with 560-570 myofibers selected per section. In all cases, the CSA measurement of each individual myofiber (technical replicates) was averaged to obtain a single biological replicate of each muscle sample.

ITRAQ analysis. A 200- $\mu$ g protein sample was precipitated with six volumes of cold acetone for $2 \mathrm{~h}$ at $-30^{\circ} \mathrm{C}$. The lysate was then centrifuged at $8,000 \times g$ for $10 \mathrm{~min}$ at $4^{\circ} \mathrm{C}(20)$. The samples were reduced, alkylated, digested, and labeled following the instructions of the ITRAQ Reagent Multi-Plex kit (AS SCIEX iTRAQ $^{\circledR}$ Reagents-Chemistry Reference Guide, P/N 4352135). The labeled peptide samples were dried in a centrifugal concentrator prior to fractionation with a High-pH Reversed-Phase Peptide fractionation kit (Thermo Fisher Scientific, Waltham, MA, USA). The peptide fractions were desalted using a C-18 Spin Column and transferred to autosampler vials. A QExactive 
Table 1. Anatomical and muscle parameters.

\begin{tabular}{lcc}
\hline \multicolumn{1}{c}{ Measurement } & Casein rats & APP rats \\
\hline Food intake $(\mathrm{g} / 7 \mathrm{~d})$ & $131.14 \pm 1.65$ & $127.98 \pm 3.39$ \\
Body weight $(\mathrm{g})$ & $223.5 \pm 2.88$ & $224.8 \pm 3.36$ \\
Soleus muscle weight $(\mathrm{mg})$ & $82.17 \pm 1.39$ & $80.97 \pm 2.05$ \\
Soleus muscle weight-to-BW $(\mathrm{mg} / \mathrm{g})$ & $0.368 \pm 0.003$ & $0.357 \pm 0.007$ \\
EDL muscle weight $(\mathrm{mg})$ & $114.36 \pm 1.74$ & $117.41 \pm 2.36$ \\
EDL muscle weight-to-BW $(\mathrm{mg} / \mathrm{g})$ & $0.512 \pm 0.005$ & $0.518 \pm 0.007$ \\
Gastrocnemius muscle weight $(\mathrm{mg})$ & $1,259.01 \pm 22.69$ & $1,365.49 \pm 24.37^{* *}$ \\
GW-to-BW ratio $(\mathrm{mg} / \mathrm{g})$ & $5.634 \pm 0.073$ & $6.075 \pm 0.062^{* *}$
\end{tabular}

BW: body weight, GW: gastrocnemius muscle weight. Values are the means \pm SE. $n=10$ per group. ${ }^{* *} p<0.01$ compared to casein-fed rats.

Orbitrap LC-MS (Thermo Fisher Scientific) was used to conduct LC-MS/MS analysis. The raw MS/MS data were converted into the MGF format using Proteome Discoverer 2.0 (Thermo Fisher Scientific). The search was conducted against the UniProt/Swiss-Prot database using the rat taxonomy filter. The identification parameters were set as follows: mass tolerance of $10 \mathrm{ppm}$ and fragment ion mass tolerance of 0.02 Da.

Ingenuity pathway analysis. Ingenuity pathway analysis (IPA; Qiagen, Hilden, Germany) was used to investigate the functional networks of the proteins expressed in the gastrocnemius muscle for the 7-d feeding experiments with casein and APP. The data used for IPA analysis, including all protein IDs $(12,886)$, were obtained from the rat taxonomy database UniProt/Swiss-Prot Accession; 3,901 analysis-ready IDs were identified after eliminating unmapped IDs and low-expression proteins (cut off: -2.0 to 2.0). These analysis-ready IDs $(3,901)$ were consolidated with the expression value set as "average" and analyzed using IPA Content version 49309495. In the signal transduction analysis using IPA, the $p$-value and activation $z$-score were calculated for each potential transcriptional regulator. The $z$-score indicated the up- or downregulation of signal transduction. The detailed procedure is described in the manual of Ingenuity Upstream Regulator Analysis in IPA (21).

Western blot analysis. SDS-PAGE analysis was conducted as described previously (22). The samples were diluted in Laemmli sample buffer and boiled for $10 \mathrm{~min}$ at $98^{\circ} \mathrm{C}$. The protein samples were separated by $10 \%$ SDS-PAGE and transferred onto PVDF membranes (Millipore, Bedford, MA, USA). The membranes were blocked for $1 \mathrm{~h}$ in Tris-buffered saline containing Tween (TBST; $20 \mathrm{mM}$ Tris-HCl [pH 7.6], $137 \mathrm{mM} \mathrm{NaCl,} \mathrm{0.1 \%}$ Tween-20) and 5\% skim milk. Thereafter, the membranes were incubated overnight at $4^{\circ} \mathrm{C}$ against Akt (\#4691, Cell Signaling Technology, Danvers, MA, USA), phospho-Akt (Ser473, \#4060, Cell Signaling Technology), mTOR (\#2972, Cell Signaling Technology), phospho-mTOR (Ser2448, \#2971, Cell Signaling Technology), p70 S6 Kinase (\#2708, Cell Signaling Technology), phospho-p70 S6 Kinase (Thr389, \#9234, Cell Signaling Technology), Fbx32/Atrogin1 (\#ab168372, Abcam, Cambridge, UK), TRIM63/MuRF1 (\#55456-1-
AP, ProteinTech, Rosemont, IL, USA), GDF8/Myostatin (\#19142-1-AP, ProteinTech), and GAPDH (\#2708, Cell Signaling Technology) with primary antibodies. The next day, the membranes were washed four times with TBST and incubated with horseradish peroxidase (HRP)-conjugated secondary antibodies (anti-rabbit or anti-mouse IgG: 1 : 1,000, Cell Signaling Technology) for $1 \mathrm{~h}$ at $23^{\circ} \mathrm{C}$. The protein bands were detected using enhanced chemiluminescence (Luminata Forte Western HRP Substrate; Merck Millipore, Bedford, MA, USA) with an ImageQuant LAS 500 imaging system (GE Healthcare, Amersham, UK). Quantification was conducted using ImageJ software (http://rsbweb.nih.gov/ ij/). To prevent mis-stripping the proteins, the expression of p-Akt and Akt and p-mTOR and mTOR were separately evaluated with the same samples.

Statistical analysis. All data are expressed as the mean \pm standard error of the mean (SE). Statistical analyses were conducted using the Student's t-test in StatView 5.0 (SAS Institute, Tokyo, Japan), and the level of significance was set at $p<0.05\left(^{*}\right)$ or $p<0.01\left(^{(*}\right)$.

\section{RESULTS}

\section{Muscle hypertrophy observed following APP feeding}

We first measured the food intake and body and skeletal muscle [soleus and EDL] weights. There were no significant differences in the above parameters when comparing casein-fed (casein rats) and APP-fed (APP rats) rats during the 7 -d feeding period (Table 1 ). In contrast, the gastrocnemius muscle weight and gastrocnemius muscle/body weight ratio of APP rats were significantly higher than those of casein rats. We then observed hypertrophic features in the gastrocnemius muscle tissues. Four major MyHC isoforms are present in the gastrocnemius muscles of mammals: three fast-twitch isoforms (MyHC IIb, MyHC IIx, MyHC IIa) and one slowtwitch isoform (MyHC I) (23). Figure S1 (Supplemental Online Material) shows muscle sections stained with antibodies against MyHC IIb, IIa, I, and IIx in green, red, blue, and black (unstained), respectively. Owing to differences in the muscle fiber compositions, we divided the gastrocnemius muscle into three analytical regions: surface lateral head gastrocnemius (LGasS), deep lateral head gastrocnemius (LGasD), and deep medial head 
gastrocnemius (MGasD). LGasS is the surface region consisting of $90 \%$ fast fibers (MyHC IIb and IIx), while LGasD and MGasD are deep regions consisting of both fast (MyHC IIb and IIx) and slow (MyHC IIa and I) fibers. The ratios of slow fibers differ between LGasD and MGasD, which are almost 50\% and over 60\%, respectively. As shown in Fig. 1 and Table 2, in the APP rats, the muscle fiber CSA in LGasS were significantly larger than those of the casein rats. The analysis of cryosections from the transverse gastrocnemius muscles revealed muscle fiber hypertrophy in LGasS (Fig. 1). The CSA of MyHC IIb-expressing myofibers increased by approximately 1.5 -fold. The data indicated that the APP rats exhibited typical muscle hypertrophy in fasttwitch MyHC isoforms (Table 2).

Proteomics analyses revealing the activation of the IGF-1 signaling pathway

We conducted ITRAQ analyses to determine differences in the expression of various skeletal muscle proteins. Over 20,000 peptides were detected and analyzed using Proteome Discoverer 2.0, and the identified pro-

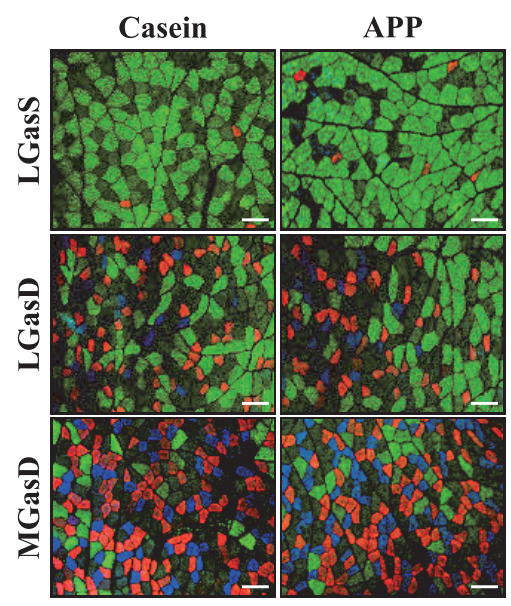

Fig. 1. Immunofluorescent images of the muscle sections stained with each $\mathrm{MyHC}$ isoform-specific antibody (green: MyHC IIb, black: MyHC IIx, red: MyHC IIa, blue: MyHC I). Results on the left and right are those for Casein and APP rats, respectively. Scale bar: $100 \mu \mathrm{m}$. teins were grouped by gene ontology. Proteins categorized into "metabolic process" were mainly detected (Fig. 2A). We then selected 86 proteins as variably expressed proteins with differences of \pm 1.5 -fold or more between the casein and APP rats. As shown in Fig. 2B, $40 \%$ of the different expressed proteins were categorized as metabolic process-regulating proteins. Ingenuity pathway analysis was also conducted to identify the activated signaling pathways, and pathways related to the proliferation and differentiation of muscle cells were observed. Hence, we observed the downregulation of transcription factor forkhead in rhabdomyosarcoma (FKHR) or FOXO signaling and upregulation of Akt/ mTOR signaling, which induces protein synthesis, cell growth, and proliferation (Fig. 2C). Akt and mTOR were identified and increased a significant (show in red), strongly predicting an increase in protein synthase (shown in orange). p70S6K was not detected, which predicted activation. The expression of phosphoinositide 3-kinase p85 (PI3Kp85) and protein phosphatase 2 (PP2A), which are related to cellular functions, was significantly decreased in APP rats (shown in green), predicting the inhibition of downstream of cell death (shown in blue). Consequently, muscle hypertrophy by APP feeding was related to increased cell proliferation and differentiation. Additionally, significant changes in IGF-1 signaling were detected (Fig. S2 (Supplemental Online Material)). Previous studies have demonstrated that exogenous IGF-1 treatment can rescue the atrophic phenotype of gastrocnemius muscle (24). In our previous study, we reported that APP intake ameliorated atrophic features during a 2 -wk limb immobilization period and increased Igf1 gene expression in soleus muscles (18). Therefore, IGF-1 signaling and/or similar pathways may be affected by APP. We then evaluated the expression of these proteins by western blotting analyses.

APP feeding augmented protein synthesis and enhanced Akt/mTOR signaling in skeletal muscle

The expression of proteins involved in the Akt/mTOR signaling pathway and ubiquitin-proteasome markers was further verified by western blotting. As shown in Fig. 3A, the phosphate Akt (p-Akt), mTOR (p-mTOR),

Table 2. Division of gastrocnemius muscle into three regions.

\begin{tabular}{|c|c|c|c|c|c|c|}
\hline & \multicolumn{6}{|c|}{ Muscle fiber cross-section area $\left(\mu \mathrm{m}^{2}\right)$} \\
\hline & \multicolumn{2}{|c|}{ LGasS } & \multicolumn{2}{|c|}{ LGasD } & \multicolumn{2}{|c|}{ MGasD } \\
\hline & Casein rats & APP rats & Casein rats & APP rats & Casein rats & APP rats \\
\hline МyHC IIb & $2,393.6 \pm 131.6$ & $3,627.0 \pm 344.0^{*}$ & $2,276.5 \pm 288.9$ & $3,188.1 \pm 225.0$ & $2,237.9 \pm 56.0$ & $3,725.0 \pm 589.6$ \\
\hline МyHc IIx & $1,473.9 \pm 111.3$ & $2,086.8 \pm 106.4^{* *}$ & $1,671.6 \pm 161.8$ & $2,276.6 \pm 77.9^{*}$ & $1,610.1 \pm 31.9$ & $1,881.3 \pm 217.3$ \\
\hline МyHC IIa & n.d. & n.d. & $1,275.9 \pm 125.6$ & $1,520.5 \pm 100.5$ & $1,352.3 \pm 139.3$ & $1,480.0 \pm 197.2$ \\
\hline MyHC I & n.d. & n.d. & $1,471.8 \pm 170.9$ & $1,883.1 \pm 96.3$ & $1,663.1 \pm 123.5$ & $2,223.2 \pm 248.2$ \\
\hline
\end{tabular}

The muscle fiber CSA was calculated, and statistical analyses were conducted. n.d.: not detected. Values are the means \pm SE. Three independent animals were used. We calculated 560-570 myofibers and averaged per animal. ${ }^{*} p<0.05,{ }^{* *} p<0.01$ compared to casein-fed rats. 
A

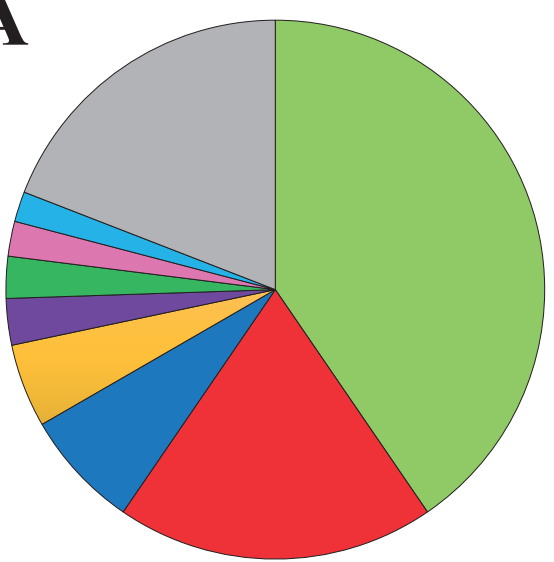

B

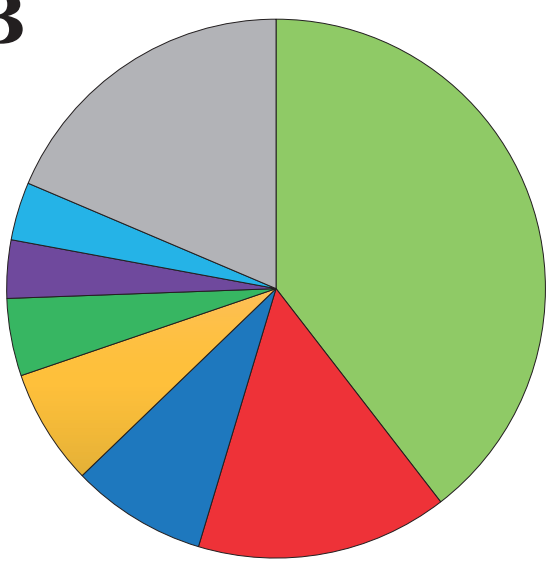

Metabolic process

Cell organization and biogenesis

Regulation of biological process

Cell differentiation

Cellular homeostasis

Transport

Cell death

Cell communication

Other

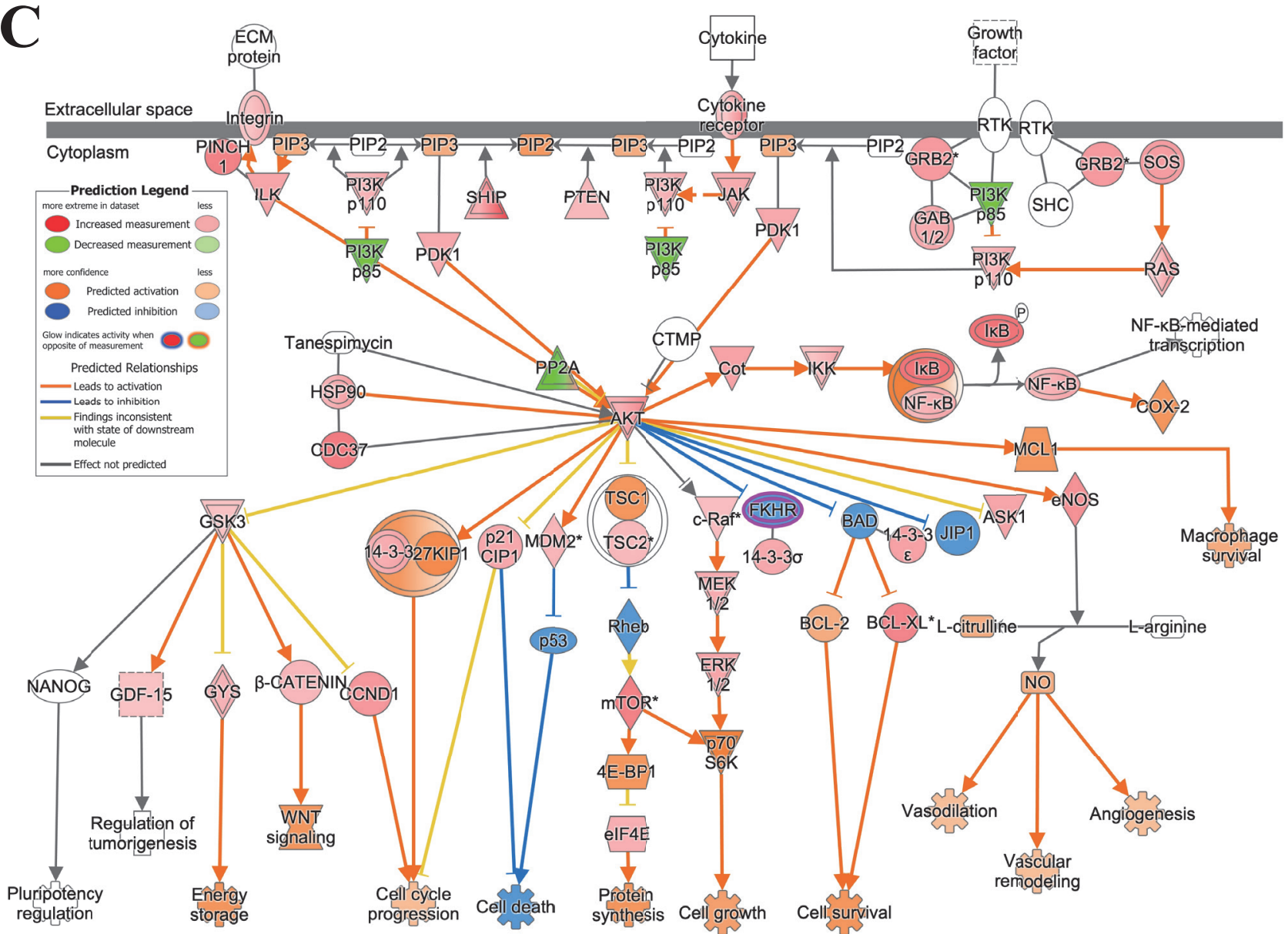

Fig. 2. Comprehensive study for elucidating the protein expression. (A) Gene ontology of proteins identified in the proteome of rat skeletal muscles. (B) Proteins exhibiting a difference of \pm 1.5 or more fold between casein and APP rats categorized by gene ontology. (C) Ingenuity pathway analysis (IPA) of PI3K/Akt signaling in rat skeletal muscle after feeding with casein or APP. Red and green indicate upregulation and downregulation compared with the corresponding parameters in casein-fed rats, respectively. Protein expression with predicted activation and inhibition is shown in orange and blue, respectively.

p70S6K (p-p70S6K), total-mTOR, and p-Akt/Akt ratio were significantly upregulated in APP rats. In contrast, there was no significant difference in the expression of Atrogin1, MuRF1, and Myostatin, which are downstream of FOXO and muscle-wasting markers $(25,26)$, between the casein and APP rats (Fig. 3B). Akt and mTOR were already identified by iTRAQ, and western blotting analyses revealed that p-Akt and p-mTOR were upregulated. p70S6K was not identified by iTRAQ; however, the phosphorylation of S6K was increased. Atrogin1, MuRF1, and Myostatin were not identified by iTRAQ, and the western blotting analyses indicated that there were no significant differences between APP and Casein. IGF-1 played a pivotal role in our model. Hence, 

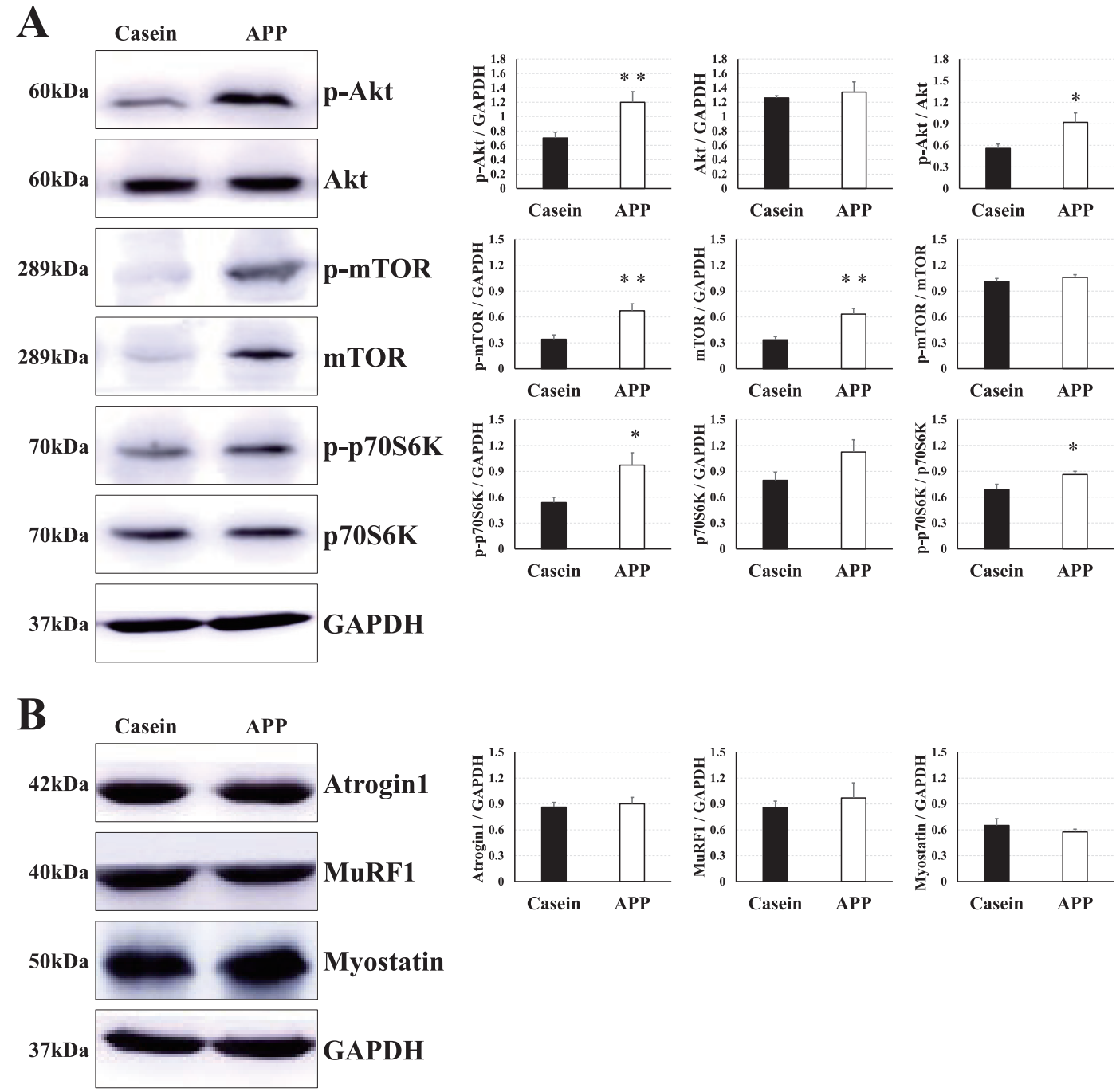

Fig. 3. Analysis of protein expression in rat skeletal muscle. Representative western blot analysis of Akt/mTOR signaling pathway markers (A) and ubiquitin-proteasome markers in gastrocnemius muscle (B). Column charts represent Akt, mTOR, p70S6K, Atrogin1, MuRF1, and Myostatin normalized by GAPDH, and the western blots represent phospho-Akt, mTOR, and p70S6K normalized by the total Akt, mTOR, and p70S6K. p-Akt: phospho-Akt, p-mTOR: phospho-mTOR, p-p70S6K: phospho-p70S6K. Data are presented as the mean \pm SE $(n=4-5) .{ }^{*} p<0.05,{ }^{* *} p<0.01$ compared to casein-fed rats. GAPDH was used as a housekeeping control.
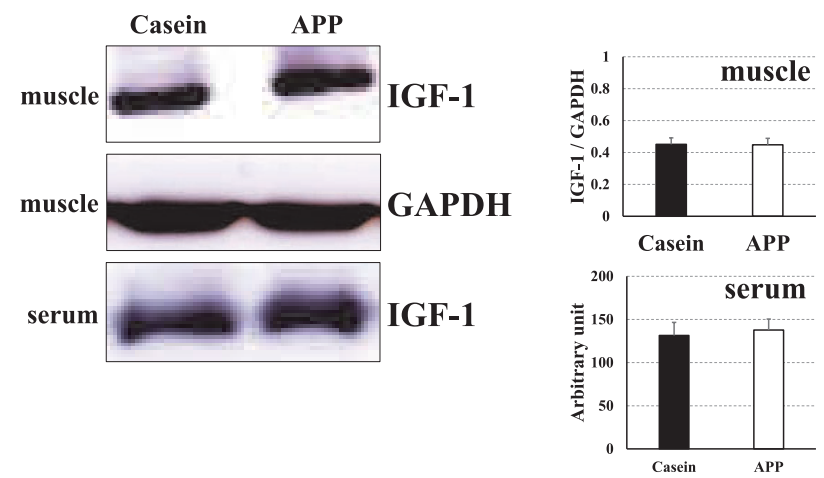

Fig. 4. Expression of IGF-1 in rat gastrocnemius muscle and serum. IGF-1 in muscle was normalized by GAPDH. The data are presented as the mean \pm SE $(n=5)$. the expression of IGF-1 was measured in the gastrocnemius muscle and serum, and there were no significant differences, as shown in Fig. 4.

\section{DISCUSSION}

Skeletal muscle is a highly malleable organ that responds to various cues, including physical activity, nutrients, and aging. For example, resistance training promotes skeletal muscle hypertrophy and improves metabolic function (27). Additionally, the surgical ablation of synergistic muscle (28-30), ladder climbing (31), and electric stimulation have also demonstrated skeletal muscle hypertrophy, mimicking resistance exercise $(32,33)$. Kobayashi et al. reported that the gastrocnemius muscle mass of a trained leg was significantly greater $(7.8 \%)$ than that of the control legs after 12 isometric resistance training sessions (34). Another previous study demonstrated that hypertrophic features require a few weeks of chronic exercise training to man- 
ifest in gastrocnemius muscle $(35,36)$. In this study, APP intake increased the gastrocnemius muscle weight within a 7-d administration period (Table 1). The period was very short, and there was no significant difference in the body weights; the ratio of gastrocnemius muscle/ body weight in APP-fed rats was greater than that in Casein-fed rats, indicating that protein synthesis may have been upregulated in the skeletal muscles.

To investigate the hypertrophic muscle features, we measured the CSA of myofibers. In our previous study, we stained gastrocnemius muscle sections with antibodies against myosin proteins specific for fast and slow fibers and measured the CSA of both fiber types (13). However, gastrocnemius muscle is composed of four major $\mathrm{MyHC}$ isoforms, which are difficult to distinguish as they exist heterogeneously within the tissues, and their compositional ratio varies between muscle types and regions (Fig. S1). In this study, we attempted to identify myofiber isoforms with hypertrophic features via immunofluorescent analysis (Fig. 1) and measured their CSA values (Table 2) in all regions (LGasS, LGasD, MGasD). Our data clearly showed that the CSA of MyHC IIb and IIx in LGasS were significantly higher in APP rats than casein rats. This means the APP feed might be a valid reason for the hypertrophy of fast fibers. The significant hypertrophy of MyHC IIx in LGasD might show the process of myofiber isoform conversion from $\mathrm{MyHC}$ IIx to MyHC IIb. Aguiar et al. reported that $5 \mathrm{wk}$ of resistance training increased the CSA by approximately 1.4-fold (37), and our model also showed an increase of almost 1.4-1.5-fold. No data regarding whether the hypertrophic features could be consistently prolonged or not were available; therefore, further studies with long-term APP feeding should be conducted.

Signaling pathways downstream of IGF-1 are required for controlling the protein synthesis and degradation balance (Fig. S2). IGF-1 signaling upregulated protein synthesis via protein kinase Akt/mTOR signaling and downregulated protein degradation by suppressing the expression of ubiquitin ligase or autophagy via FOXO signaling (14). Resistance exercise-induced muscle hypertrophy is associated with the upregulation of anabolic signaling pathways (p-Akt, p-mTOR, p-p70S6K), and also results in a reduced rate of autophagy with a decrease in the LC3-II/I ratio and an increase in the level of p62/sequestosome 1 (38). Kwon et al. reported that $8 \mathrm{wk}$ of resistance training could result in a 3.2-fold increase in muscle mass and muscular strength (38). Additionally, they also reported the enhancement of the activity of anabolism-related proteins (Akt, mTOR, p70S6K). In this study, we used iTRAQ-based proteome and western blotting analyses to examine the differences in protein expression due to APP feeding (Figs. 2 and 3). As shown in Fig. 3, APP promoted skeletal muscle hypertrophy by activating the Akt/mTOR signaling pathway to enhance protein synthesis rather than inhibit protein degradation, and the underlying mechanism was very similar to that governing resistance training. Although IGF-1 expression remained unaltered (Fig. 4), downstream Akt/mTOR signaling was upregulated by APP. Other signaling factors that altered Akt/mTOR signaling without IGF-1 may exist. For example, a previous study also identified that mechanical loading can induce muscle hypertrophy and activate Akt signaling without the involvement of the IGF-1 receptor $(39,40)$, such as insulin receptor sand $\mathrm{Ca}^{2+}(41)$. The expression of these factors in our models and the experiments using mTOR inhibitors will be evaluated in future studies to investigate factors that affect muscle hypertrophy.

\section{Authorship}

Research conception and design: KU, TK, MF, and NGI; experiments: MM, MF, KK, and NGI; statistical analysis of the data: MM, TM, and NGI; interpretation of the data: MM, MF, TM, and NGI; writing of the manuscript: MM, EY, MF, TM and NGI.

\section{Disclosure of state of COI}

This study was funded by Nippon Suisan Kaisha, Ltd. Eriko Yoshida and Kenji Uchida are current employees of Nippon Suisan Kaisha, Ltd.

\section{Acknowledgments}

This study was supported in part by JSPS KAKENHI Grant Number 21H03330, "Nanotechnology Platform Project” operated by the Ministry of Education, Culture, Sports, Science and Technology (MEXT), Japan (No. JPMXP09 S19NM0048). We would like to thank Editage (www.editage.com) for English language editing.

\section{Supporting information}

Supplemental online material is available on J-STAGE.

\section{REFERENCES}

1) Iannuzzi-Sucich M, Prestwood KM, Kenny AM. 2002. Prevalence of sarcopenia and predictors of skeletal muscle mass in healthy, older men and women. J Gerontol A Biol Sci Med Sci 57: M772-777.

2) Marcell TJ. 2003. Sarcopenia: causes, consequences, and preventions. J Gerontol A Biol Sci Med Sci 58: M911-916.

3) Faulkner JA, Larkin LM, Claflin DR, Brooks SV. 2007. Age-related changes in the structure and function of skeletal muscles. Clin Exp Pharmacol Physiol 34: 10911096.

4) Cai X, Zhu C, Xu Y, Jing Y, Yuan Y, Wang L, Wang S, Zhu X, Gao P, Zhang Y, Jiang Q, Shu G. 2016. Alpha-ketoglutarate promotes skeletal muscle hypertrophy and protein synthesis through Akt/mTOR signaling pathways. Sci Rep 6: 26802.

5) Dijk FJ, van Dijk M, Walrand S, van Loon LJC, van Norren K, Luiking YC. 2018. Differential effects of leucine and leucine-enriched whey protein on skeletal muscle protein synthesis in aged mice. Clin Nutr ESPEN 24: 127-133.

6) Ohno Y, Ando K, Ito T, Suda Y, Matsui Y, Oyama A, Kaneko H, Yokoyama S, Egawa T, Goto K. 2019. Lactate stimulates a potential for hypertrophy and regeneration of mouse skeletal muscle. Nutrients 11: 867.

7) Lim CH, Gil JH, Quan H, Viet DH, Kim CK. 2018. Effect 
of 8-week leucine supplementation and resistance exercise training on muscle hypertrophy and satellite cell activation in rats. Physiol Rep 6: e13725.

8) Oertzen-Hagemann V, Kirmse M, Eggers B, Pfeiffer K, Marcus K, de Marees M, Platen P. 2019. Effects of 12 weeks of hypertrophy resistance exercise training combined with collagen peptide supplementation on the skeletal muscle proteome in recreationally active men. Nutrients 11: 1072.

9) Dort J, Leblanc N, Maltais-Giguere J, Liaset B, Cote CH, Jacques H. 2013. Beneficial effects of cod protein on inflammatory cell accumulation in rat skeletal muscle after injury are driven by its high levels of arginine, glycine, taurine and lysine. PLoS One 8: e77274.

10) Ayabe T, Mizushige T, Ota W, Kawabata F, Hayamizu K, Han L, Tsuji T, Kanamoto R, Ohinata K. 2015. A novel Alaska pollack-derived peptide, which increases glucose uptake in skeletal muscle cells, lowers the blood glucose level in diabetic mice. Food Funct 6: 2749-2757.

11) Mizushige T, Kawabata F, Uozumi K, Tsuji T, Kishida T, Ebihara K. 2010. Fast-twitch muscle hypertrophy partly induces lipid accumulation inhibition with Alaska pollack protein intake in rats. Biomed Res 31: 347-352.

12) Kawabata F, Mizushige T, Uozumi K, Hayamizu K, Han L, Tsuji T, Kishida T. 2015. Fish protein intake induces fast-muscle hypertrophy and reduces liver lipids and serum glucose levels in rats. Biosci Biotechnol Biochem 79: 109-116.

13) Morisasa M, Goto-Inoue N, Sato T, Machida K, Fujitani M, Kishida T, Uchida K, Mori T. 2019. Investigation of the lipid changes that occur in hypertrophic muscle due to fish protein-feeding using mass spectrometry imaging. J Oleo Sci 68: 141-148.

14) Schiaffino S, Mammucari C. 2011. Regulation of skeletal muscle growth by the IGF1-Akt/PKB pathway: insights from genetic models. Skelet Muscle 1: 4.

15) Milan G, Romanello V, Pescatore F, Armani A, Paik JH, Frasson L, Seydel A, Zhao J, Abraham R, Goldberg AL, Blaauw B, DePinho RA, Sandri M. 2015. Regulation of autophagy and the ubiquitin-proteasome system by the FoxO transcriptional network during muscle atrophy. Nat Commun 6: 6670.

16) Wagatsuma A, Shiozuka M, Takayama Y, Hoshino T, Mabuchi K, Matsuda R. 2016. Effects of ageing on expression of the muscle-specific E3 ubiquitin ligases and Akt-dependent regulation of Foxo transcription factors in skeletal muscle. Mol Cell Biochem 412: 59-72.

17) Kang SH, Lee HA, Kim M, Lee E, Sohn UD, Kim I. 2017. Forkhead box $\mathrm{O} 3$ plays a role in skeletal muscle atrophy through expression of E3 ubiquitin ligases MuRF-1 and atrogin-1 in Cushing's syndrome. Am J Physiol Endocrinol Metab 312: E495-E507.

18) Fujitani M, Mizushige T, Kawabata F, Uozumi K, Yasui M, Hayamizu K, Uchida K, Okada S, Keshab B, Kishida T. 2019. Dietary Alaska pollack protein improves skeletal muscle weight recovery after immobilization-induced atrophy in rats. PLoS One 14: e0217917.

19) Bloemberg D, Quadrilatero J. 2012. Rapid determination of myosin heavy chain expression in rat, mouse, and human skeletal muscle using multicolor immunofluorescence analysis. PLoS One 7: e35273.

20) Fu L, Xu Y, Hou Y, Qi X, Zhou L, Liu H, Luan Y, Jing L, Miao Y, Zhao S, Liu H, Li X. 2017. Proteomic analysis indicates that mitochondrial energy metabolism in skeletal muscle tissue is negatively correlated with feed effi- ciency in pigs. Sci Rep 7: 45291.

21) Mori T, Kitani Y, Hatakeyama D, Machida K, Goto-Inoue N, Hayakawa S, Yamamoto N, Kashiwagi K, Kashiwagi A. 2020. Predation threats for a 24-h period activated the extension of axons in the brains of Xenopus tadpoles. Sci Rep 10: 11737.

22) Laemmli UK. 1970. Cleavage of structural proteins during the assembly of the head of bacteriophage T4. Nature 227: 680-685.

23) Sawano S, Komiya Y, Ichitsubo R, Ohkawa Y, Nakamura M, Tatsumi R, Ikeuchi Y, Mizunoya W. 2016. A one-step immunostaining method to visualize rodent muscle fiber type within a single specimen. PLoS One 11: e0166080.

24) Song YH, Song JL, Delafontaine P, Godard MP. 2013. The therapeutic potential of IGF-I in skeletal muscle repair. Trends Endocrinol Metab 24: 310-319.

25) Mammucari C, Milan G, Romanello V, Masiero E, Rudolf R, Del Piccolo P, Burden SJ, Di Lisi R, Sandri C, Zhao J, Goldberg AL, Schiaffino S, Sandri M. 2007. FoxO3 controls autophagy in skeletal muscle in vivo. Cell Metab 6: 458-471.

26) Rodriguez J, Vernus B, Chelh I, Cassar-Malek I, Gabillard JC, Hadj Sassi A, Seiliez I, Picard B, Bonnieu A. 2014. Myostatin and the skeletal muscle atrophy and hypertrophy signaling pathways. Cell Mol Life Sci 71: 43614371.

27) Williams MA, Haskell WL, Ades PA, Amsterdam EA, Bittner V, Franklin BA, Gulanick M, Laing ST, Stewart KJ, American Heart Association Council on Clinical Cardiology, American Heart Association Council on Nutrition Physical Activity and Metabolism. 2007. Resistance exercise in individuals with and without cardiovascular disease: 2007 update: a scientific statement from the American Heart Association Council on Clinical Cardiology and Council on Nutrition, Physical Activity, and Metabolism. Circulation 116: 572-584.

28) Goodman CA, Frey JW, Mabrey DM, Jacobs BL, Lincoln HC, You JS, Hornberger TA. 2011. The role of skeletal muscle mTOR in the regulation of mechanical load-induced growth. J Physiol 589: 5485-5501.

29) McCarthy JJ, Mula J, Miyazaki M, Erfani R, Garrison K, Farooqui AB, Srikuea R, Lawson BA, Grimes B, Keller C, Van Zant G, Campbell KS, Esser KA, Dupont-Versteegden EE, Peterson CA. 2011. Effective fiber hypertrophy in satellite cell-depleted skeletal muscle. Development 138: 3657-3666.

30) Akpulat U, Onbasilar I, Kocaefe YC. 2016. Tenotomy immobilization as a model to investigate skeletal muscle fibrosis (with emphasis on Secreted frizzled-related protein 2). Physiol Genomics 48: 397-408.

31) Lee S, Barton ER, Sweeney HL, Farrar RP. 2004. Viral expression of insulin-like growth factor-I enhances muscle hypertrophy in resistance-trained rats. J Appl Physiol (1985) 96: 1097-1104.

32) Wong TS, Booth FW. 1990. Protein metabolism in rat tibialis anterior muscle after stimulated chronic eccentric exercise. J Appl Physiol (1985) 69: 1718-1724.

33) Ogasawara R, Arihara Y, Takegaki J, Nakazato K, Ishii N. 2017. Relationship between exercise volume and muscle protein synthesis in a rat model of resistance exercise. J Appl Physiol 123: 710-716.

34) Kobayashi K, Ogasawara R, Tsutaki A, Lee K, Ochi E, Nakazato K. 2012. Genetic strain-dependent protein metabolism and muscle hypertrophy under chronic iso- 
metric training in rat gastrocnemius muscle. Physiol Res 61: $527-535$.

35) Soffe Z, Radley-Crabb HG, McMahon C, Grounds MD, Shavlakadze T. 2016. Effects of loaded voluntary wheel exercise on performance and muscle hypertrophy in young and old male C57Bl/6J mice. Scand J Med Sci Sports 26: 172-188.

36) Petriz BA, Gomes CP, Almeida JA, de Oliveira GP Jr, Ribeiro FM, Pereira RW, Franco OL. 2017. The effects of acute and chronic exercise on skeletal muscle proteome. J Cell Physiol 232: 257-269.

37) Aguiar AF, de Souza RW, Aguiar DH, Aguiar RC, Vechetti IJ Jr, Dal-Pai-Silva M. 2011. Creatine does not promote hypertrophy in skeletal muscle in supplemented compared with nonsupplemented rats subjected to a similar workload. Nutr Res 31: 652-657.

38) Kwon I, Jang Y, Cho JY, Jang YC, Lee Y. 2018. Long-term resistance exercise-induced muscular hypertrophy is associated with autophagy modulation in rats. J Physiol Sci 68: 269-280.

39) Spangenburg EE, Le Roith D, Ward CW, Bodine SC. 2008. A functional insulin-like growth factor receptor is not necessary for load-induced skeletal muscle hypertrophy. J Physiol 586: 283-291.

40) Matheny RW, Merritt E, Zannikos SV, Farrar RP, Adamo ML. 2009. Serum IGF-I-deficiency does not prevent compensatory skeletal muscle hypertrophy in resistance exercise. Exp Biol Med (Maywood) 234: 164-170.

41) Rommel C, Bodine SC, Clarke BA, Rossman R, Nunez L, Stitt TN, Yancopoulos GD, Glass DJ. 2001. Mediation of IGF-1-induced skeletal myotube hypertrophy by PI(3)K/ Akt/mTOR, PI(3)K/Akt/GSK3 pathways. Nat Cell Biol 3: 1009-1013. 